\title{
Exergetic analysis of compressor characteristics using computer modeling
}

\author{
Alexeev Gennady Valentinovich \\ Department of Process and Equipment in Food Production \\ St. Petersburg National Research University of Information \\ Technology, Mechanics and Optics \\ St. Petersburg, Russia \\ gva2003@mail.ru
}

Savelev Aleksey Petrovich

Department of Process and Equipment in Food Production St. Petersburg National Research University of Information Technology, Mechanics and Optics

St. Petersburg, Russia

savelevaleksey1983@gmail.com

\author{
Romanchikov Sergey Aleksandrovich \\ Doctoral student \\ Military academy of logistics \\ St. Petersburg, Russia \\ romanchkovspb@mail.ru
}

\author{
Bridenko Igor Iosifovich \\ Department of Process and Equipment in Food Production \\ St. Petersburg National Research University of Information \\ Technology, Mechanics and Optics \\ St. Petersburg, Russia \\ bridenko@mail.ru
}

\begin{abstract}
Modern requirements for resource saving seriously raise the issues of secondary use of basic raw materials and auxiliary materials in the production cycle. Food production, in addition, uses water in significant quantities and as an auxiliary material. Unconditional superiority in such processes is occupied by different stages of washing the processed raw materials and transporting waste. The paper describes the assessment of the efficiency of water recycling.
\end{abstract}

After primary purification in physical filters, the averaged wastewater, free of fatty inclusions, gets into the aerotank, where it is cleaned up under the influence of microorganisms. At this stage of purification, water is saturated with oxygen, which is a source of energy for microorganisms. In an oxygen environment with a sufficient volume of nutrients and a corresponding temperature, aerobic organisms begin to multiply actively, forming macrocolonies. These colonies are active silt, precipitating as a sediment and circulating in the water column during aeration. In the aeration tank, the water is purified by oxidizing the organic matter and then absorbing it. Aeration sinks are diverted to the soot, where the active sludge settles and is redirected through the water exchange system to the anaerobic treatment site or to repeated aeration.

Keywords - resource-saving, secondary use of auxiliary materials, food production, washing of processed raw materials, waste transportation, evaluation of work efficiency, aerotank.

\section{INTRODUCTION}

However, costs should be considered when choosing a cleaning system. Aerotanks are volatile devices and their operation is impossible when there is no power supply. Microorganisms that purify sewage are killed in less than 12 hours. Therefore, with in-creased power consumption, it becomes necessary to connect a cleaning plant to a backup net-work or a generator capable of ensuring uninterrupted operation of the compressor. Depending on the manufacturer and a model of the treatment plant, aerotanks may differ in terms of techno-logical and design principles. Most domestic companies that produce domestic and industrial installations use pneumatic compressors for aeration of effluents entering the tank. Aerators are made of ceramics, metal or polymeric materials. There is a combined aeration system, where the air injected by the compressor, exits through the nozzle and is directed to the filter plate, which increases the air distribution area and reduces the bubble size. Such technology has found wide application in imported domestic installations and some domestic models. The operation of such systems is impossible without the participation of various kinds of air blowers, in particular fans. The design of these devices is highly dependent on the geometry of the individual working elements, for example, blades. The authors propose an approach for working out such details with the use of computer modeling of the implemented processes.

Compressor technology nowadays is used not only for environmental protection equipment, but also for a wide range of areas of economy. With its help, air masses are injected into various mines during the extraction of minerals, oil wells, as well as powerful ventilation of production premises or movement of special products in food or biotechnological industries. It is known that the force interaction of gas or liquid flow with a streamlined body is carried out through two types of force reactions: through the action of distributed forces of excess pressure and through the action on the body of tangential friction forces. In some cases, the design of compressors, which are the main component of injection systems for the purpose of energy-saving of their experimental development, is using the models of these devices $[1,2,3]$ 
Calculation practice shows that practically used compressors in the range of large Reynolds numbers, the total force of the aerodynamic action of the blades of the rotating rotor are determined mainly by the force from distributed pressure. Only with zero lift for very thin wing profiles, the component from frictional forces begins to be significant in the overall strength of the resistance. Proceeding from this, it is advisable for the non-fine profile of the injection blade to determine the force action created exclusively by the distributed pressure forces [4].

\section{CARRIED OUT RESEARCH AND DISCUSSION OF RESULTS}

For the refined model of a heated object, let us use a sphere to which thermal impulses are periodically fed from friction against abrasive sections of the working organs of the process equipment that occurs in the thermal process.

Experimental definition of distribution of pressure on such structure can be made at a purge in a subsonic wind tunnel rectangular, using the blade, with a constant structure in all cross-sections. On the average section, the blade is drained. Each aperture of a drainage on the blade is hermetically connected with the top of a tube battery manometer. In blow covered with airflow on a wing, some distribution of pressure is established. Some accuracy depends on the number of points of a drainage, cleanliness of processing of reception apertures, etc. It is possible to define this distribution by gauging of pressure in all points of a drainage [5].

If, at the same time, a positive excess pressure is set in the hole on the wing, the liquid level in the manometer tube drops below the zero mark; If the excess pressure is negative (rarefaction in this part of the wing), the liquid level rises. Denoting the height of the change in the liquid level in the tube of the manometer and considering it to be an algebraic value $(\mathrm{h}>0$, if the liquid in the tube drops, and $\mathrm{h}<0$, if the liquid rises), it is possible for each measured value to find an overpressure:

$$
\Delta p_{i}=p_{i}-p_{\infty}=\gamma_{\delta} \Delta h_{i}
$$

where $\gamma_{\delta}$ - specific gravity of liquid in manometer (if manometer is filled with colored water, $\gamma_{\delta}=10-3 \mathrm{~kg} / \mathrm{cm}^{3}$ ).

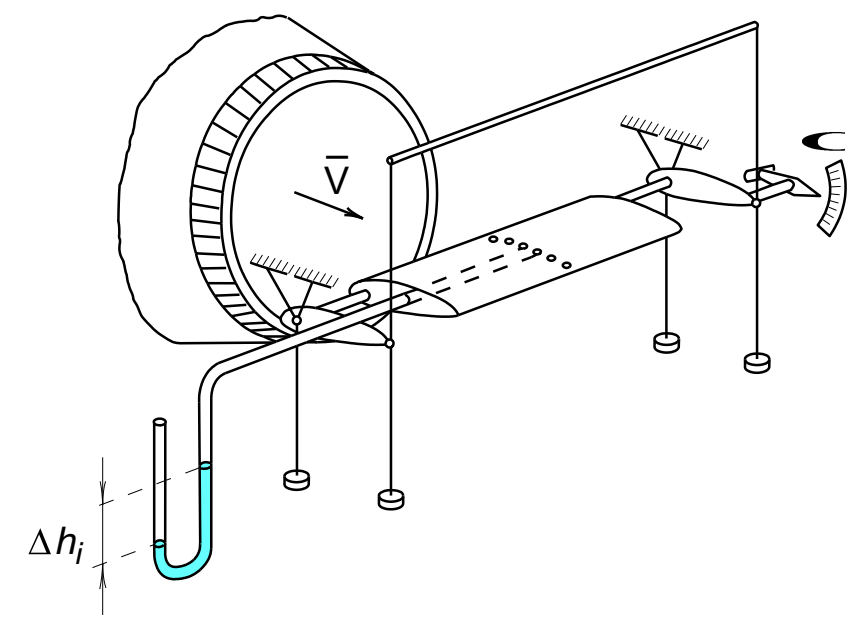

Fig. 1. Overpressure measuring circuit

Simultaneously with the fixation of pressure at various points in the profile, the velocity head must be measured, using the Pitot tube. Then the pressure coefficient in the hole on the profile is:

$$
c_{p i}=\frac{p_{i}-p_{\infty}}{\rho_{\infty} \frac{v_{\infty}^{2}}{2}}=\frac{\gamma_{\delta} \Delta h_{i}}{\gamma_{\mathrm{p}} \Delta h_{\text {pito }}}
$$

Using the obtained data on the pressure distribution, it is possible to construct the so-called vector diagram, Fig. 2. On the vector diagram, the blade is plotted accurately, and the drainage points are drawn; at each point, a vector is equal to the magnitude of the pressure coefficient at $c_{p_{i}}$, a given point is attached to the wing surface. If the coefficient $c_{p_{i}}$ is positive, then the vector is directed to the wing; if negative, then the vector is directed to the outside. The ends (or beginnings) of a vector are connected by an envelope line. Such vector diagram gives a graphically clear picture of the distribution of the rarefaction and compression zones along the profile of the blade profile [6].

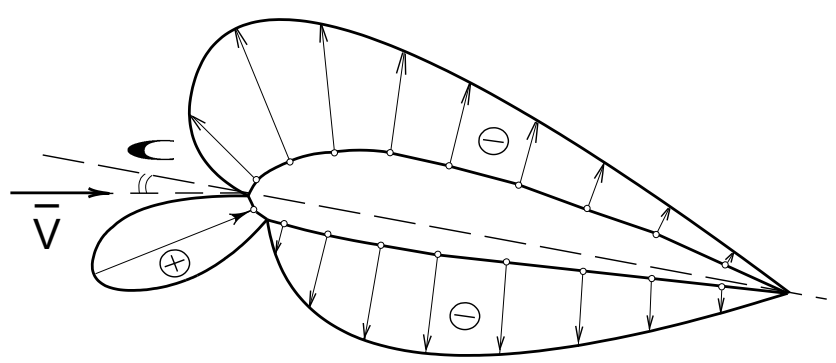

Fig. 2. The vector diagram of the blade 
The axis $0 x_{1}$ is in parallel to a vector of not indignant speed of an accumulating stream. The angle between axes $0 x$ and $0 x_{1}$ is angle of attack $\alpha$.

On a contour of a considered structure, we shall note that the bottom surface covered $O B A$ and top surface - $O C A$. On these surfaces, points $B$ and $C$ are special. These points are mostly removed from a chord of the blade accordingly on the bottom and top surfaces of the blade and allocate a frontal surface covered $B O C$ and a fodder surface $B A C$.

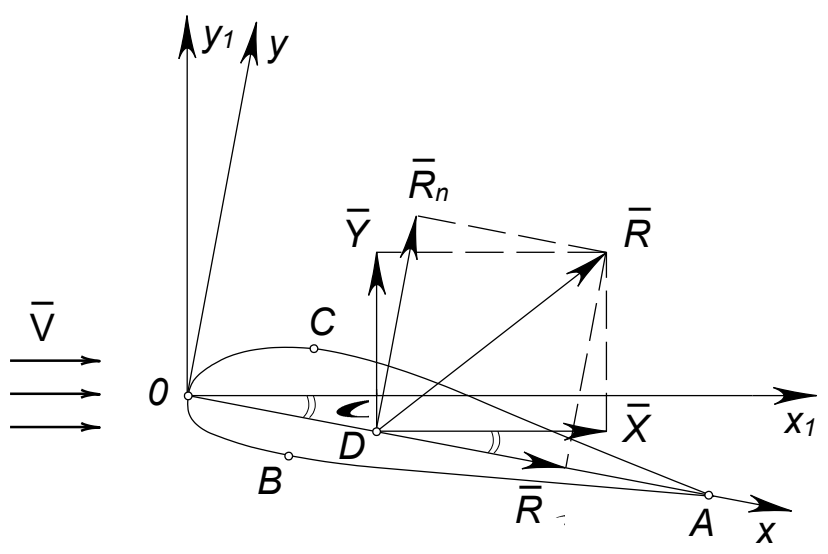

Fig. 3. The diagram of the appendix to a structure of the blade of various forces

In fig. 3, point $D$ notes the center of pressure in which equally effective aerodynamic force $\vec{R}$ is enclosed. Aerodynamic force $\vec{R}$ can be spread out on two mutually perpendicular components. If these components are parallel to axes of high-speed coordinate system, component $X$ refers to as a force of frontal resistance, and $Y$ - elevating force. If these components are parallel to axes of the associated coordinate system, component $R_{\tau}$ refers to the longitudinal force, $R_{\Pi}$ - cross-section. Let us consider a surface of the blade of individual scope. On this blade it is possible to allocate an elementary rectangular platform $d S$, formed with an element of a contour $d S$ so $d S=d s \cdot 1$. The elementary piece of a contour $d S$ is inclined to axis $0 x$ at angle $\theta$ (fig. 4).

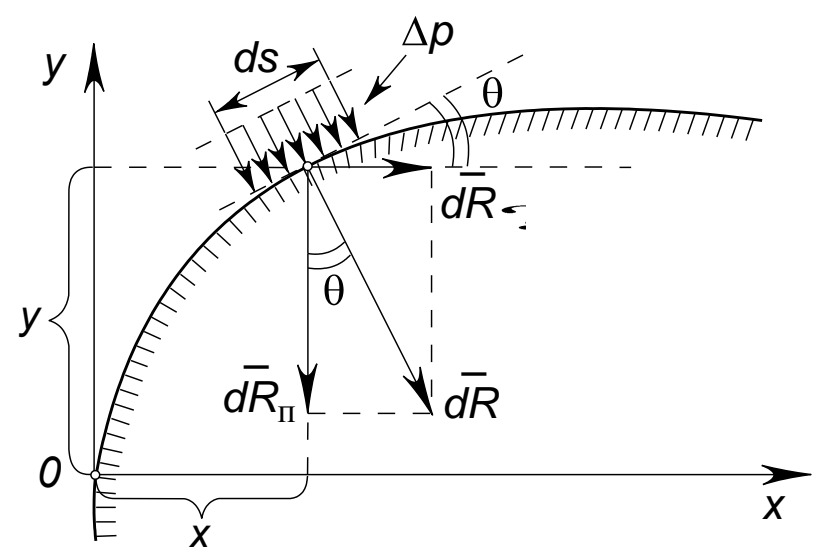

Fig. 4. Occurrence of horizontal and vertical components

If on a surface of a streamline structure in this seat, superfluous pressure $\Delta p$ force is enclosed to the given site $d R$ where $d R=\Delta p d s$ operates on a normal to a surface. This force can be spread out on two mutually perpendicular components $d R_{\tau}$ and $d R_{\mathrm{\Pi}}$, parallel to axes $0 x$ and $0 y$ :

$$
\begin{gathered}
d R_{\tau}=d R \sin \theta=\Delta p \sin \theta d s ; \\
d R_{\Pi}=-d R \cos \theta=-\Delta p \cos \theta d s
\end{gathered}
$$

From fig.4 let us see that $d s \cos \theta=d x ; d s \sin \theta=d y$. Then:

$$
d R_{\tau}=\Delta p d y ; d R_{\mathrm{\Pi}}=-\Delta p d x .
$$

Being based on dependences (3), we shall find total forces: longitudinal $R_{\tau}$, parallel to chord $O A$, and cross-section $R_{\text {п }}$, perpendicular to a chord.

For reception of force $R_{\text {п }}$, it is necessary to integrate dependence for $d R_{\mathrm{\Pi}}$ on top and the bottom surface of a structure:

$$
R_{\text {п }}=-\int_{y_{O}}^{x_{4}} \Delta p_{\mathrm{B}} d x+\int_{y_{O}}^{x_{4}} \Delta p_{H} d x
$$

where $\Delta p_{\text {в }}$ and $\Delta p_{\text {н }- \text { superfluous pressure accordingly }}$ on the top and bottom surface of a structure. Considering that $x_{o}=0 ; x_{\mathrm{A}}=b$; let us have:

$$
R_{\text {п }}=\int_{0}^{b}\left(\Delta p_{\text {н }}-\Delta p_{\text {в }}\right) d x
$$


In a similar way for reception of force $R_{\tau}$, it is necessary to integrate dependence from (3) on frontal (site $B O C$ ) and fodder (site $B A C$ ) to parts of a structure:

$$
R_{\tau}=\int_{y_{B}}^{y_{C}} \Delta p_{\text {л }} d y_{1}-\int_{y_{B}}^{y_{C}} \Delta p_{\mathrm{\kappa}} d y,
$$

Let us notice that:

$$
y_{B}=y_{\min } ; \quad y_{C}=y_{\max }(5)
$$

Then:

$$
R_{\tau}=\int_{y_{\min }}^{y_{\max }}\left(\Delta p_{л}-\Delta p_{\kappa}\right) d y
$$

Let us pass from longitudinal and cross-section forces $R_{\tau}$ and $R_{\Pi}$ to forces in a high-speed system of coordinates: to force of frontal resistance $X$ and elevating force $Y$. This transition is carried out under formulas [7]:

$$
\begin{gathered}
X=R_{\tau} \cos \alpha+R_{\text {п }} \sin \alpha \\
Y=-R_{\tau} \sin \alpha+R_{\text {п }} \cos \alpha
\end{gathered}
$$

Forces $X$ and $Y$ in aerodynamics are represented through factors of frontal resistance $c_{x}$ and elevating force $c_{y}$ under formulas:

$$
X=c_{x} \frac{1}{2} \rho_{\infty} v_{\infty}^{2} S \quad Y=c_{y} \frac{1}{2} \rho_{\infty} v_{\infty}^{2} S
$$

Here the characteristic area $S$ maybe presented as $S=1 \cdot b$ (the blade of individual scope).

In a similar way, longitudinal and cross-section forces can be presented:

$$
R_{\tau}=c_{\tau} \frac{\rho_{\infty} v_{\infty}^{2}}{2} S \quad R_{\Pi}=c_{\Pi} \frac{\rho_{\infty} v_{\infty}^{2}}{2} S
$$

Substituting (8) and (9) in (7), it is possible to receive:

$$
\begin{gathered}
c_{x}=c_{\tau} \cos \alpha+c_{\text {п }} \sin \alpha ; \\
c_{y}=-c_{\tau} \sin \alpha+c_{\Pi} \cos \alpha,
\end{gathered}
$$

Dimensionless factors $c_{\tau}$ and $c_{n}$ also are defined, if (9) one substitutes in parities (4) and (6) and considers a definition of factor of pressure under the formula (2):

$$
\begin{gathered}
c_{\text {п }}=\frac{1}{b} \int_{0}^{b}\left(c_{p_{H}}-c_{p_{B}}\right) d x \\
c_{\tau}=\frac{1}{b} \int_{y_{\min }}^{y_{\max }}\left(c_{p_{\text {л }}}-c_{p_{\mathrm{\kappa}}}\right) d y
\end{gathered}
$$

It is meaningful to enter dimensionless coordinates:

$$
\bar{x}=\frac{x}{b} ; \quad \bar{y}=\frac{y}{b}
$$

Let us enter also new designations:

$$
\begin{gathered}
\Delta c_{p_{\text {п }}}=c_{p_{\text {н }}}-c_{p_{\text {в }}} ; \\
\Delta c_{p_{\tau}}=c_{p_{\text {л }}}-c_{p_{\text {к }}}
\end{gathered}
$$

Let us come to following rated formulas:

$$
\begin{gathered}
c_{\text {п }}=\int_{0}^{1} \Delta c_{p_{\text {п }}} d \bar{x} \\
c_{\tau}=\int_{\bar{y}_{\min }}^{\bar{y}_{\max }} \Delta c_{p_{\tau}} d y
\end{gathered}
$$

Integrating this dependence on a wing contour, let us get the dependence [8]:

$$
M_{z}=\frac{1}{2} \Delta \Delta p \cdot d\left(x^{2}\right)+\oint \Delta p d\left(y^{2}\right) .
$$

Contour integrals in (15) are calculated at a contour round clockwise.

In an aerodynamics, the moment of pitch of $M_{z}$ is bound to coefficient of the tangent moment $c_{m z}$ with formula:

$$
M_{z}=c_{m z} \frac{\rho_{\infty} v_{\infty}^{2}}{2} S b
$$

where $S=b \cdot 1$

Comparing records (15) and (16) and considering designation of a coefficient of pressure (2), let us receive: 


$$
c_{m_{z}}=\frac{1}{2} \cdot \frac{1}{b^{2}}\left\lceil c_{p} d\left(x^{2}\right)+\oint c_{p} d\left(y^{2}\right)^{-} .\right.
$$

Let us transform one of contour integrals:

$$
J_{x}=\oint c_{p} d\left(x^{2}\right)=\int_{0}^{b} c_{p_{B}} d x^{2}+\int_{b}^{0} c_{p_{H}} d x^{2}=\int_{0}^{b}\left(c_{p_{B}}-c_{p_{H}}\right) d x^{t}
$$

Similary:

$$
J_{y}=\oint c_{p} d y^{2}=\int_{y_{\min }}^{y_{\max }} c_{p_{\text {л }}} d y^{2}-\int_{y_{\max }}^{y_{\min }} c_{p_{\mathrm{k}}} d y^{2}=\int_{y_{\min }}^{y_{\max }}\left(c_{p_{\pi}}-c_{p_{\mathrm{K}}}\right) d y^{2}
$$

Considering received and also designations (12) and (13), instead of (17), let us write:

$$
c_{m_{z}}=0,5\left(J_{x}+J_{y}\right)
$$

where:

$$
J_{\bar{x}}=-\int_{0}^{1} \Delta c_{p_{\mathrm{n}}} d\left((\bar{x})^{2}\right) \quad J_{\bar{y}}=\int_{\bar{y}_{\min }}^{\bar{y}_{\max }} \Delta c_{p_{\tau}} d\left(\bar{y}^{2}\right)
$$

Tangage moment can be shown with forces $\vec{X}$ and $\vec{Y}$, attached to a center of pressure in point $D$ with coordinates $x_{D}=x_{\text {д } ;} y_{D}=0$ :

$$
M_{z}=-X x_{\text {д }} \sin \alpha-Y x_{\text {д }} \cos \alpha,
$$

Let us transform:

$$
\bar{x}_{\text {д }}=\frac{x_{\text {д }}}{b}=-\frac{M_{z}}{Y \cos \alpha+X \sin \alpha},
$$

or:

$$
\bar{x}_{\text {д }}=-\frac{c_{m_{z}}}{c_{y} \cos \alpha+c_{x} \sin \alpha},
$$
[9].

This formula defines the provision of a center of pressure

An inspection of the explained reasons was carried out by means of the virtual model operation of a wind tunnel of subsonic speeds. The blade model in the model is suspended on extensions in a working part of a pipe with realization of the following conditions:

to install the blade at the given angle of attack $\alpha$; to place the receiver of a tube of Pitot-Prandtl in a zone of a nonperturbed stream before the model;

to measure size $\Delta h_{\text {п }}$ and to put a pipe into operation;

to measure change from the position of equilibrium of fluid levels in pipes of the battery manometer and to enter measured sizes $\Delta h_{i}$ with their signs in the table;

to provide accuracy of measurements to the tenth shares of centimeter.

After carrying out measurements on a formula (2), values of a coefficient of pressure $c_{p_{i}}$ are calculated and results are entered in the table.

Then let us build a coordinate charts of $c_{p}=f(\bar{x})$ and $c_{p}=f_{1}(\bar{y})$. For this purpose, using these tables, the experimental points are $\left(c_{p_{i}}, \bar{x}_{i}\right)$ and $\left(c_{p_{i}}, \bar{y}_{i}\right)$. These points connect the smooth curves. With inscriptions $c_{p_{\text {н }} \text { and }} c_{p_{\mathrm{s}}}$, let us put pressure distribution curve on the top and bottom surface of a wing (see fig. 5); on other chart curve, pressure profiles on front and fodder parts of a profile are noted by inscriptions $c_{p_{\mathrm{\pi}} \text { and }} c_{p_{\mathrm{k}}}$.

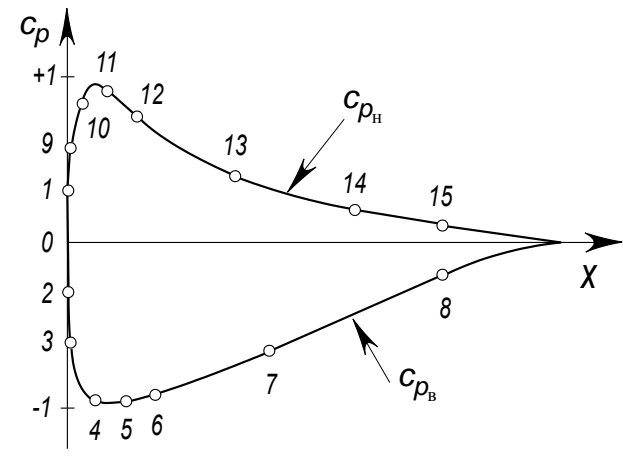

Fig. 5. Coordinate charts

Further it is necessary to calculate the value of coefficient $c_{\text {п }}$ on the first formula from (14) For this purpose let us use Simpson's formula for a numerical integration:

$$
\begin{aligned}
c_{\mathrm{n}} & =\frac{1}{3(N-1)} \Delta c_{p_{\mathrm{n}}}^{(1)}+4 c_{p_{\mathrm{n}}}^{(2)}+\Delta c_{p_{\mathrm{n}}}^{(4)}+\ldots+\Delta c_{p_{\mathrm{n}}}^{(N-1)}- \\
& +2 \mathbf{d} c_{p_{\mathrm{n}}}^{(3)}+\Delta c_{p_{\mathrm{n}}}^{(5)}+\ldots+\Delta c_{p_{\mathrm{n}}}^{(N-2)} \pm \Delta c_{p_{\mathrm{n}}}^{(N)}
\end{aligned}
$$

For use of this formula, all intervals of change $\bar{x}(0 \leq x \leq 1)$ drop to $N-1$ equal parts, where $N-$ odd number. Any $\Delta c_{p_{\mathrm{n}}}^{(i)}$ is $\Delta c_{p_{\mathrm{n}}}=c_{p_{\mathrm{H}}}-c_{p_{\mathrm{B}}}$ in a point with coordinates $\bar{x}=\bar{x}_{i}=\frac{i-1}{N-1}$. Practical calculation of value 
$\Delta c_{p_{\text {п }}}^{(i)}$

$\Delta p_{\text {п }}$ acts from the schedule measurement of distance between curves $c_{p_{\mathrm{H}}}$ and $c_{p_{\mathrm{B}}}$ with the corresponding coordinate $\bar{x}$ which take algebraic sign $\Delta c_{p_{\text {п }}}^{(i)}$. Value $\Delta c_{p_{n}}$ in extreme points, apparently from fig. 5 , is always equal to zero: $\Delta c_{p_{\text {п }}}^{(1)}=\Delta c_{p_{\text {п }}}^{(N)}=0$.

The similar pattern of calculation of integral can be offered also for the second dependence (14). The calculated formula takes a form [10]:

$$
\begin{aligned}
& c_{\tau}=\frac{\bar{y}_{\max }-\bar{y}_{\text {min }}}{3\left(N_{1}-1\right)} \boldsymbol{c}_{p_{\tau}}^{(1)}+4 \lambda c_{p_{\tau}}^{(2)}+\Delta c_{p_{\tau}}^{(4)}+\ldots+\Delta c_{p_{\tau}}^{\left(N_{1}-1\right)} \underline{-} \\
& +2 \Delta c_{p_{\tau}}^{(3)}+\Delta c_{p_{\tau}}^{(5)}+\ldots+\Delta c_{p_{\tau}}^{\left(N_{1}-2\right)} \underline{-}+\Delta c_{p_{\tau}}^{\left(N_{1}\right)}
\end{aligned}
$$
with coordinate:

$$
\bar{y}=\bar{y}_{\max }-\frac{\bar{y}_{\max }-\bar{y}_{\min }}{N_{1}-1}(i-1)
$$

These value can be defined by immediate measurement of the distance between curves $c_{p_{\mathrm{r}}}$ and $c_{p_{\mathrm{\kappa}}}$ at the corresponding coordinate $\bar{y}$. Also the sign of size has to be considered $\Delta c_{p_{\tau}}$. Here, as well as at calculation of value $c_{n}, \mathrm{r} N_{1}-$ odd number, also $\Delta c_{p_{\tau}}^{(1)}=\Delta c_{p_{\tau}}^{\left(N_{1}\right)}=0$.

The calculated values $c_{n}$ also allow one to find aerodynamic coefficients $c_{x}$ and $c_{y}$ according to formulas (10).

For calculation of coefficient of the tangage moment $c_{m_{z}}$ on a formula (18), let us calculate integrals $J_{\bar{x}}$ and $J_{\bar{y}}$, set by formulas (19). For this purpose let us use the same technique of a numerical integration, as with calculation of coefficients $c_{\text {п } \text { and }} c_{\tau}$. Dependences have to be for this purpose graphically constructed $c_{p}$ by $\bar{x}_{2}$ and $c_{p}$ by $\bar{y}_{2 \text {. Having }}$ broken an integration interval $0 \leq x^{2} \leq 1$ on an even number of pieces, with use of a formula of Simpson, let us calculate integral $J_{\bar{x}}$, and then similarly $J_{\bar{y}}$. On a formula (20), it is possible to find the provision of a center of pressure $\bar{x}_{\text {д }}$.

\section{THE RESULTS OF THE EXPERIMENT}

The virtual experiment for definition of aerodynamic characteristics of a profile of the blade was made on the model containing the wind tunnel of subsonic speeds forming an airflow and a receiver pipe for utilization of an airflow.

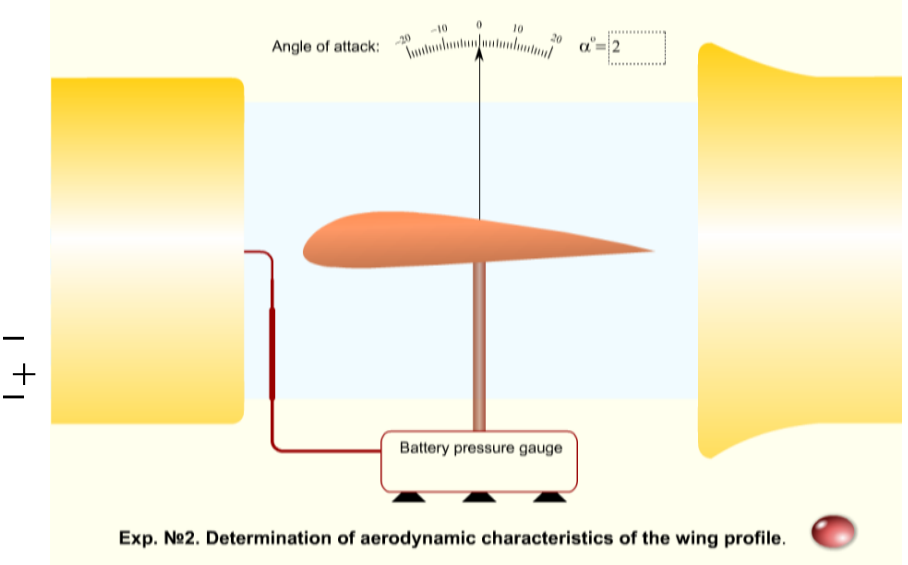

Fig. 6. The virtual model of laboratory installation

In a working gap of a pipe, the blade with a number of drainage openings on a profile is installed. Each opening is connected to the measuring manometer (a piezometer with water). All manometers are united in the battery. Besides, Pitot-Prandtl's tube is connected to the battery manometer. The tube is established just before the blade in a nonperturbed part of a stream.

The angle of attack of a wing was changed ranging from $14^{\circ}$ to $+14^{\circ}$ with a step of 1 lake. At the same time, indications of all 15 piezometers connected to the corresponding drainage openings of the blade changed.

Geometrical measurements were taken expressing measuring rulers on an axis " $\mathrm{X}$ " and " $\mathrm{Y}$ ". Rulers are moved by means of a mouse.

The click in the field of the battery manometer is called the panel for measurement of fluid levels in the corresponding piezometers. Measurements were taken by a measuring ruler on an axis of "Y", in the mode chosen and providing necessary accuracy.

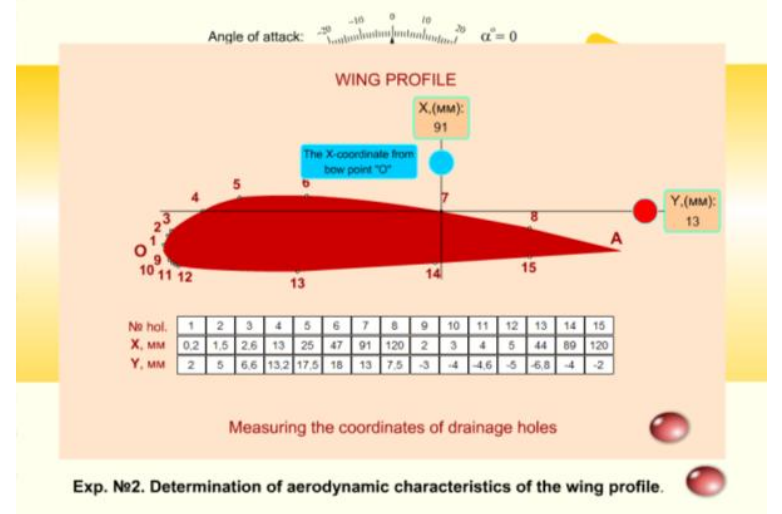

a)

Fig.7. Carrying out measurements on the model 


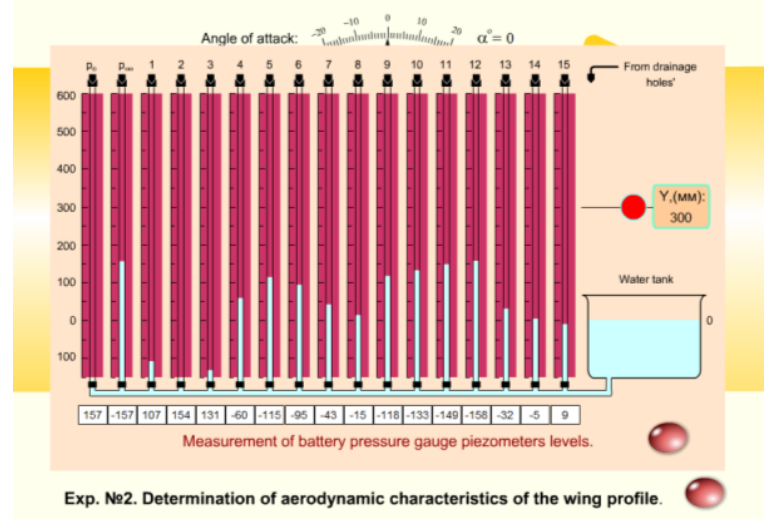

b)

Figure 7, cont.

\section{CONCLUSIONS}

The conducted pilot studies showed good convergence with the results received on natural laboratory installations saving electric power 6-8 times. It allows one to investigate more carefully characteristics of compressors to choose the option that is more preferable in the relation. Data interpretation was carried out by means of a software package "Mathcad" according to the known aerodynamic model of a wing not of a thin profile which is written down in a look:

$$
J_{x}=\oint c_{p} d\left(x^{2}\right)=\int_{0}^{b} c_{p_{B}} d x^{2}+\int_{b}^{0} c_{p_{H}} d x^{2}=\int_{0}^{b}\left(c_{p_{B}}-c_{p_{H}}\right) d x^{2}
$$

$$
J_{y}=\oint c_{p} d y^{2}=\int_{y_{\min }}^{y_{\max }} c_{p_{\mathrm{n}}} d y^{2}-\int_{y_{\max }}^{y_{\min }} c_{p_{\mathrm{k}}} d y^{2}=\int_{y_{\min }}^{y_{\max }}\left(c_{p_{\mathrm{n}}}-c_{p_{\mathrm{\kappa}}}\right) d y^{2}
$$

The error of the obtained data made $15-17 \%$ in comparison with calculated results on analytical model that testifies to a possibility of preliminary estimate of new designs of compressors by the used technique.

\section{References}

[1] B. E. Ryabchikov, Modern treatment, M.: Delhi, plus, 2013, pp. 680.

[2] F. R. Spellman, Handbook on cleaning natural and sewage. Water supply and Sewerage. Translation from English, SPb.: TSOP "the professions", 2014, pp. 1312.

[3] V.V. Solodyannikov, Calculation and mathematical modeling of processes of water treatment, M, Energoatomizdat, 2003.

[4] G I. Petrov, High-speed and space research. Of swear works, M.: Nauka, 1992, pp. 306.

[5] A.N. Osiptsov, "Slim profile in the flow of the dispersed blend," Izv. USSR Academy of Sciences, MGH, Vol. 5, pp. 147-154, 1981.

[6] I.I. Halavin, E.V. Kravtsova, A.G. LEU, Mathematical modeling opportunity resource. In the collection: Integrating science, society, production, and industry, Collection of articles of International scientific-practical conference, 2016, pp. 52-54.

[7] G.V. Alekseev, I.I. Bridenko, Virtual laboratory workshop on the course "FLUID MECHANICS", St.-Petersburg, 2007, pp. 198.

[8] V.B. Baranov, Fluid Mechanics and gas dynamics, M.: Izd-vo MGU, Moscow, 1987, pp. 184.

[9] G.V. Alekseev, N. Mosina, Abrasive processing of potatoes and vegetables with the discreteness of the energy supply. Monograph, Saratov, 2013, pp. 215.

[10] I.I. Halavin, I.V. Novikov, A.G. LEU, evaluation of the effectiveness of prior training in formulating starch. In the book: Innovativetechnological development of science, Collection of articles of international scientific-practical conference in 3 parts, 2017, pp. 162166 